\title{
Brain abscess in Korean children: A 15-year single center study
}

Cha Gon Lee, M.D.', Seong Hun Kang, M.D.', Yae Jean Kim, M.D. ${ }^{1}$, Hyung Jin Shin, M.D. ${ }^{2}$, Hyun Shin Choi, M.D.', Jee Hun Lee, M.D. ${ }^{1}$ and Mun Hyang Lee, M.D.

Department of Pediatrics', Department of Neurosurgery ${ }^{2}$, School of Medicine, Sungkyunkwan University, Samsung Medical Center, Seoul, Korea

Received: 15 September 2009, Revised: 2 November 2009 Accepted: 1 December 2009

Corresponding Author: Yae-Jean Kim, M.D.

Department of Pediatrics, School of Medicine, Sungkyunkwan University, Samsung Medical Center, 50 Irwon-dong, GangnamGu, Seoul 135-710, Korea

Tel: +82.2-3410-3539, Fax: +82.2-3410-0043

E-mail: aejeankim@skku.edu

Copyright () 2010 by The Korean Pediatric Society
Purpose: A brain abscess is a serious disease of the central nerve system. We conducted this study to summarize the clinical manifestations and outcomes of brain abscesses.

Methods: A retrospective chart review of pediatric patients diagnosed with brain abscesses from November 1994 to June 2009 was performed at Samsung Medical Center, Seoul, Korea.

Results: Twenty-five patients were included in this study. On average, 1.67 cases per year were identified and the median age was 4.3 years. The common presenting clinical manifestations were fever $(18 / 25,72 \%)$, seizure $(12 / 25,48 \%)$, altered mental status $(11 / 25,44 \%)$, and signs of increased intracranial pressure $(9 / 25$, $36 \%)$. A total of $14(56 \%)$ patients had underlying illnesses, with congenital heart disease $(8 / 25,32 \%)$ as the most common cause. Predisposing factors were identified in 15 patients $(60 \%)$. The common predisposing factors were otogenic infection (3/25, 12\%) and penetrating head trauma $(3 / 25,12 \%)$. Causative organisms were identified in $64 \%$ of patients (16/25). The causative agents were $S$. intermedius $(\mathrm{n}=3), S$. aureus $(\mathrm{n}=3), S$. pneumoniae $(\mathrm{n}=1)$, Group B streptococcus $(n=2), E$. coli $(n=1)$, P. aeruginosa $(n=1)$, and suspected fungal infection $(n=5)$. Seven patients received medical treatment only while the other 18 patients also required surgical intervention. The overall fatality rate was $16 \%$ and $20 \%$ of patients had neurologic sequelae. There was no statistical association between outcomes and the factors studied.

Conclusion: Although uncommon, a brain abscess is a serious disease. A high level of suspicion is very important for early diagnosis and to prevent serious consequences.

Key words: Brain, Abscess, Child, Korea

This is an open-access article distributed under the terms of the Creative Commons Attribution Non-Commercial License (http://creativecommons.org/licenses/by$\mathrm{nc} / 3.0 /$ ) which permits unrestricted non-commercial use, distribution, and reproduction in any medium, provided the original work is properly cited.

\section{Introduction}

A brain abscess is a focal collection of infectious material within the brain, which can arise as a complication from a variety of causes including infection, trauma, and surgery. A wide variety of organisms (bacteria, fungi, protozoa, and parasites) can cause abscess 
formation. Brain abscesses occur relatively infrequently because of the abundant blood supply to the brain and the protection of the brain by the blood-brain barrier. In the pre-antibiotics era when medicines with good central nervous system (CNS) penetration were not available, brain abscesses were almost uniformly lethal ${ }^{1)}$. Despite the major advances in neuroimaging and the increased availability of potent antibiotics and neurosurgical intervention, the incidence of brain abscesses has not changed much. Brain abscesses still have high rates of neurologic impairment and fatality ${ }^{2)}$. While a number of reports have reviewed brain abscesses in adult populations, only a few comprehensive reports on long follow-ups of pediatric cases are available ${ }^{2-8)}$. In this study, we analyzed data from 25 patients with brain abscesses and we describe the clinical features of these brain abscesses.

\section{Materials and methods}

Pediatric patients less than 19 years old who were diagnosed with a brain abscess at Samsung Medical Center, Seoul, Korea from November 1994 through June 2009 were included. We searched all the patients whose diagnosis at discharge was brain abscess(es) using Samsung Medical Information System (SMIS) and only included cases with radiological evidence; 21 patients had brain parenchymal abscesses, 3 patients had a subdural abscess, and 1 patient had an epidural abscess. Clinical data (clinical manifestations, initial laboratory findings, causative organisms, characteristics of abscesses, treatment modalities, and outcomes) were collected based on retrospective chart reviews. Statistical analysis, such as univariate analysis, Mann-Whitney test, Fisher's exact test, was performed to evaluate association between outcomes and the factors studied.

\section{Results}

\section{Patient characteristics}

A total of 25 pediatric patients were diagnosed with one or more brain abscesses during 14.7 consecutive years, an average of 1.67 cases per year. Fourteen patients were male and 11 patients were female (ratio 1.3:1). The median age was 4.3 years (range, 0.1-18.9 years), and the abscesses occurred most commonly in patients younger than 2 years of age, including 1 neonate $(11 / 25,44 \%)$. Five of these 11 patients (45\%) were born prematurely $(<37$ weeks gestational age).

Underlying diseases and predisposing factors are listed in Table 1. Fourteen patients (56\%) presented with known underlying medical conditions: congenital heart disease (CHD, $\mathrm{n}=8)$, immunodeficiency $(\mathrm{n}=3)$ due to acute myeloblastic leukemia (AML), myelodysplastic syndrome (MDS) and agammaglobulinaemia, and suspected arterial venous malformation $(\mathrm{n}=1)$. In patients younger than 2 years old $(\mathrm{n}=11)$, prematurity was also considered to be an underlying medical condition ( $\mathrm{n}=5)$. Two patients had both prematurity and CHD and one patient had MDS and CHD. Predisposing factors were identified in 15 patients $(15 / 25,60 \%)$. The common predisposing factors were otogenic infection $(\mathrm{n}=3)$ and penetrating head trauma $(n=3)$ followed by preceding meningitis $(n=2)$, neurosurgical procedures $(\mathrm{n}=2)$, endocarditis $(\mathrm{n}=2)$, ventriculoperitoneal shunts $(\mathrm{n}=2)$, and poor oral hygiene with lymphangioma at the tongue base $(\mathrm{n}=1)$.

\section{Clinical presentation}

The most common presenting symptom was fever (17/25, 68\%). The median body temperature at diagnosis was $38.5^{\circ} \mathrm{C}$ (range, $36.3-41^{\circ} \mathrm{C}$ ). Twelve patients ( $48 \%$ ) had seizures (generalized or partial). Altered mental status (ranging from drowsy to coma) was observed in 12 patients (48\%): 7 patients were drowsy, 2 were in a stupor, and 3 were in a semicoma. One patient's mental status could not be assessed due to deep sedation. The median interval between symptom onset and diagnosis by imaging studies was 11 days in 23 patients (range, 1-119 days). Two patients did not have any symptoms. One patient had the longest time delay of 119 days until diagnosis. This patient was a 9 month-old female who had been born prematurely (gestational age $30+1 / 7$ weeks) at an outside hospital and was transferred to our hospital under the diagnosis of cystic encephalomalacia, ventriculitis, and parenchymal abscess with shunt infection.

The Glasgow coma scale was only evaluated in six patients $(24 \%)$ at diagnosis; severe brain injury (GCS $\leq 8)$ was diagnosed in two, moderate brain injury (GCS 9-12) in one, and minor brain injury (GCS $\geq 13$ ) in three patients. Focal neurologic deficits were observed in seven patients (28\%). Signs of increased intracranial pressure (IICP) such as vomiting, neck stiffness, or bulged fontanelle were observed in nine patients (36\%).

\section{Laboratory data}

The median WBC count at diagnosis ( $\mathrm{n}=24$ patients) was $11,740 / \mu \mathrm{L}$ (range, $580-43,660 / \mu \mathrm{L}$ ), the median level of C-reactive protein $(\mathrm{n}=24)$ was $1.69 \mathrm{mg} / \mathrm{dL}$ (range, $0.06-29.06 \mathrm{mg} / \mathrm{dL}$ ), and the median erythrocyte sedimentation rate $(\mathrm{n}=18)$ was $23 \mathrm{~mm} /$ hr (range, 8-105 mm/hr). Lumbar puncture was performed in 14 patients (56\%); the median CSF WBC was 206/ $\mu \mathrm{L}$ (range, 0 $15,700 / \mu \mathrm{L}$ ); the median protein level was $158 \mathrm{mg} / \mathrm{dL}$ (range, 10 - 
1,502), and the median glucose level was $53 \mathrm{mg} / \mathrm{dL}$ (range, 2-85 $\mathrm{mg} / \mathrm{dL})$.

\section{Microbiological study}

Microbiological diagnosis was confirmed in $64 \%$ of patients (16/25 patients) based on abscess cultures ( $n=5 / 13)$, blood cultures $(n=6 / 21)$, CSF cultures $(n=4 / 12)$, vegetation culture by open-heart surgery $(n=1 / 2)$, or pathology reports $(n=2 / 2)$. Streptococcus intermedius $(\mathrm{n}=3)$, Staphylococcus aureus $(\mathrm{n}=3)$, Group B Streptococcus $(\mathrm{n}=2)$, Streptococcus pneumoniae $(\mathrm{n}=1)$, Escherichia coli $(\mathrm{n}=1)$, Pseudomonas aeruginosa $(\mathrm{n}=1)$, Aspergillus spp. (Aspergillus flavus, Aspergillus spp.) ( $\mathrm{n}=2)$, Candidia albicans $(\mathrm{n}=2)$, and a suspected fungal infection $(\mathrm{n}=1)$ were detected. Two patients had the same pathogens in both blood and CSF cultures: Streptococcus pneumonia and Pseudomonas aeruginosa. Staphylococcus intermedius ( $\mathrm{n}=3$ ), Staphylococcus aureus $(\mathrm{n}=1)$, and Aspergillus flavus ( $\mathrm{n}=1)$ were present in abscess cultures. Candia albicans was identified in a vegetation culture of one patient. The organisms present in two patients were confirmed by abscess pathology reports only; an Aspergillus species in one patient and a suspected fungal infection (no species identification) in the other patient.

\section{Radiologic study}

For radiologic diagnosis, computer tomography (CT, $\mathrm{n}=17$ ), magnetic resonance (MRI, $n=5$ ), or ultrasonography (US, $n=3$ ) images were obtained. Twenty-one patients were diagnosed with a brain parenchymal abscess, 3 patients with a subdural abscess, and 1 patient with an epidural abscess. Among 21 patients with brain parenchymal abscess, a single lesion was observed in 9 patients (43\%) and multiple lesions were observed in 12 patients (57\%). The distribution of single lesions was similar in both sides of the brain; five patients had abscesses in the right hemisphere and four had abscesses in the left hemisphere. The most common location of single abscesses was the temporal lobe $(3 / 9,33 \%)$ followed by the parietal lobe $(2 / 9,22 \%)$, frontoparietal lobe $(1 / 9,11 \%)$, basal ganglia $(1 / 9,11 \%)$, cerebellum $(1 / 9,11 \%)$, and occipital lobe $(1 / 9$, $11 \%)$.

\section{Treatment and outcomes}

All patients received combination antimicrobial therapy. Seven

Table 1. Characteristics and Outcomes of Brain Abscesses in 25 Pediatric Patients

\begin{tabular}{|c|c|c|c|c|c|}
\hline \multirow{2}{*}{ Patients } & \multirow{2}{*}{ Sex } & \multirow{2}{*}{ Age (years) } & \multirow{2}{*}{ Underlying diseases/predisposing factors } & \multicolumn{2}{|r|}{ Outcome } \\
\hline & & & & Mortality & Neurologic impairment \\
\hline 1 & $\mathrm{~F}$ & 0.8 & Preterm(GA 30+1/7 wks), VP shunt infection & Dead & NA \\
\hline 2 & M & 1.3 & Preterm (GA $35+2 / 7$ wks), poor oral hygiene due to tongue base lymphangioma & Alive & None \\
\hline 3 & $\mathrm{~F}$ & 1.3 & $\begin{array}{l}\text { Preterm ( } 36 \text { wks), bicuspid aortic valve with aortic regurgitation, pneumonia sepsis with } \\
\text { DIC and ICH }\end{array}$ & Alive & None \\
\hline 4 & M & 3.2 & Tricuspid atresia & Alive & None \\
\hline 5 & $\mathrm{~F}$ & 4.8 & Head trauma & Alive & Dysarthria, limping gait \\
\hline 6 & $\mathrm{~F}$ & 5.0 & Ventricular septal defect & Alive & None \\
\hline 7 & $\mathrm{~F}$ & 5.0 & Medulloblastoma, neurosurgery & Dead & NA \\
\hline 8 & $\mathrm{~F}$ & 5.3 & $\mathrm{R} / \mathrm{O} \mathrm{AVM}, \mathrm{ICH}$, neurosurgery & Alive & Right hemiplegia \\
\hline 9 & M & 7.7 & Pulmonary atresia & Dead & NA \\
\hline 10 & M & 7.9 & MDS, patent foramen ovale, & Alive & None \\
\hline 11 & M & 8.4 & Pulmonary stenosis with atrial septal defect & Alive & None \\
\hline 12 & M & 15.5 & Preceding dental procedure, endocarditis & Alive & None \\
\hline 13 & M & 17.2 & Lt COM with cholesteatoma & Alive & None \\
\hline 14 & M & 17.6 & No underlying disease or predisposing factor & Alive & Hemianopsia \\
\hline 15 & $\mathrm{~F}$ & 18.9 & Rt COM with cholesteatoma & Alive & None \\
\hline 16 & M & 0.8 & Agammaglobulinema & Alive & None \\
\hline 17 & $\mathrm{~F}$ & 13.1 & Head trauma & NA & NA (lost to follow- up) \\
\hline 18 & M & 0.1 & Patent foramen ovale, fungal endocarditis & Alive & None \\
\hline 19 & $\mathrm{~F}$ & 0.1 & Preceding meningitis & Alive & Cerebral palsy \\
\hline 20 & M & 1.7 & AML & Alive & None \\
\hline 21 & M & 4.3 & $\mathrm{AOM}$ & Alive & None \\
\hline 22 & M & 0.1 & Preterm (GA 25+3/7 wks), patent ductus arteriosus, ICH & Dead & NA \\
\hline 23 & $\mathrm{~F}$ & 1.3 & Head trauma & Alive & Epilepsy \\
\hline 24 & M & 0.1 & Preceding meningitis & Alive & None \\
\hline 25 & $\mathrm{~F}$ & 1.0 & Preterm (GA 28 wks), VP shunt infection & Alive & None \\
\hline
\end{tabular}

Abbreviations: MDS, myelodysplastic syndrome; AOM, acute otitis media; DIC, disseminated intravascular coagulation syndrome; AML, acute myeloblastic leukemia; COM, chronic otitis media; AVM; arterio-venous malformation; ICH, intra-cerebral hemorrhage; VP shunt, ventriculoperitoneal shunt; NA, not applicable 
patients received medical treatment only and 18 patients received additional surgical interventions (17 neurosurgeries and 2 openheart surgeries for vegetation removal). One patient had two neurosurgeries. Commonly performed neurosurgical procedures were bur hole drainage $(n=9)$, surgical excision with open craniotomy $(\mathrm{n}=4)$, and image-guided stereotactic aspiration $(\mathrm{n}=3)$. The median duration of antimicrobial therapy was 42 days (range, 12-108 days). Commonly used antimicrobials were vancomycin (23/24, 96\%), third-generation cephalosporin (19/24, 76\%), metronidazole (13/24, 52\%), and amphotericin B (5/24, 21\%).

Four patients died and the overall fatality rate was 16\% (4/25 patients). One patient was lost to follow-up. The median followup duration was 3.1 years in the remaining 20 patients (range, 0.2-13.2). Neurological impairments such as epilepsy, motor impairment (limping gait, hemiplegia), cerebral palsy, visual field defect (hemianopsia), were observed in 5 of 20 survivors (Table 1).

There was no statistical association between outcomes (fatality and neurological impairments) and the factors studied; age, sex, days from symptom onset to diagnosis, presence of congenital heart diseases, initial mental status, seizure at diagnosis, IICP at diagnosis, focal neurologic defect at diagnosis, initial body temperature, WBC counts, level of CRP, diagnostic images used (CT vs US vs MRI), and treatments used (medical treatment only vs surgery combined).

\section{Discussion}

Brain abscesses are serious and life-threatening lesions of the CNS. They are uncommon in pediatric patients and most of the published literature comprises case reports. This study is one of the few to report comprehensive data on brain abscesses collected from 25 pediatric patients at a single tertiary center during a period of 14.7 years.

At the University of Virginia Children's Hospital between 2000-2007, an average of 1.5 children per year were admitted to the inpatient pediatric service with a primary diagnosis of brain abscess $^{4}$. Lee et $\mathrm{al}^{2(4)}$ reported 27 pediatric cases of Korean children over 10 years, an average of 2.7 cases per year. In our study, an average of 1.67 pediatric patients per year were identified during 14.7 consecutive years. In terms of age distribution, brain abscesses occurred most commonly in patients younger than 2 years of age.

The clinical manifestations of brain abscesses initially tend to be nonspecific and there are no typical laboratory or CSF study results, which often causes a delay in diagnosis ${ }^{9}{ }^{9}$. The median interval from symptom onset to diagnosis was 11 days in our study, similar to another report $(13 \text { days })^{11}$. Previously, headache was reported as the most common symptom of a brain abscess in nonpediatric patients ${ }^{12-14)}$. In our study, fever was the most common presenting symptom. However, it should also be emphasized that fever is a common symptom of many other childhood illnesses ${ }^{15}$, and as many as $32 \%$ of children did not have high fever. Therefore, fever is not always a reliable indicator of a brain abscess in children.

Seydoux et $\mathrm{al}^{16)}$ observed focal neurologic deficits in $50 \%$ of their patients; these deficits generally occurred days to weeks after the onset of headache. They also reported that $25 \%$ of patients developed seizures and that having a seizure was the first manifestation of a brain abscess ${ }^{16)}$. Lee et $\mathrm{al}^{24)}$ reported that $38 \%$ of their survivors had neurological sequlae. In our study, seizure was present in $48 \%$ of cases and focal neurologic deficits in $28 \%$ of cases. Neurological impairment was observed in 5 of 20 survivors with available follow-up data. However, not all patients had followup visits to our institution or long-term follow-ups of the same duration, and some patients' neurological conditions were not described in detail. Therefore, it is possible that the actual rate of neurological impairment in our study population could have been higher than what was observed.

In non-pediatric patients, the most commonly underlying diseases are diabetes mellitus and/or liver cirrhosis ${ }^{12}$. In pediatric patients, congenital heart disease has been reported to be the leading condition ${ }^{2)}$. In our study, congenital heart disease was also the leading condition. Among 11 babies less than 2 years old, prematurity was the most common underlying condition $(\mathrm{n}=5)$ and congenital heart disease was the second most common underlying condition $(\mathrm{n}=3)$. Two patients were premature babies and also had congenital heart disease and intra-cerebral hemorrhage.

Radiology tests (CT or MRI) are tests of choice for the diagnosis and monitoring of brain abscesses ${ }^{14)}$. In particular, MRI is more sensitive for detecting early cerebritis and estimates the extent of central necrosis more accurately than the other test modalities. CT scanning is not as sensitive as MRI for the diagnosis of brain abscesses, but is more easily performed in an emergency-room setting ${ }^{14)}$. Ultrasongraphy is also a possible neuroimaging modality in infants who have an open fontanelle and sutures ${ }^{17)}$. In addition, ultrasonography is a readily accessible and portable neuroimaging modality. Therefore, ultrasonography is very useful in pediatric patients. In our study, all three types of radiologic tests were used to diagnose brain abscesses. CT was the most commonly-used imaging modality (68\% of patients) followed by MRI (16\%) and ultrasonography (12\%). Because most of patients were evaluated in the emergency room or the patient's condition was critical at the time of work-up for brain abscesses, CT was more frequently used than MRI in our study population. 
Published fatality rates for brain abscesses range from 0 to $30 \%$ $10,16,18-20,24,25)$. In this study, the overall fatality was $16 \%$. Several studies have failed to find an association between outcomes and the factors studied such as age, focal neurologic deficits, seizures, laboratory findings, causative organisms, characteristics of abscesses, and treatment modalities, ${ }^{1,1,21-23)}$. In this study, there was no statistical association between outcomes and the factors studied.

Brain abscesses result in significant morbidity and high fatality. Because brain abscesses can have subtle initial presentations, a high level of suspicion is very important for early diagnosis in pediatric patients, particularly those with underlying conditions such as congenital heart disease or prematurity with a complicated postnatal course.

\section{References}

1) Tseng JH, Tseng MY. Brain abscess in 142 patients: factors influencing outcome and mortality. Surg Neurol 2006;65:557-62.

2) Goodkin HP, Harper MB, Pomeroy SL. Intracerebral abscess in children: historical trends at Children's Hospital Boston. Pediatrics 2004;113:176570.

3) Sato T, Kaneda M, Ichikawa M, Suzuki D, Nakagawa A, Kobayashi R. Current approaches to management of cerebral fungal infection in pediatric patients with hematologic disorders. J Pediatr Hematol Oncol 2008;30:249-53.

4) Sheehan JP, Jane JA, Ray DK, Goodkin HP. Brain abscess in children. Neurosurg Focus 2008;24:E6.

5) Frazier JL, Ahn ES, Jallo GI. Management of brain abscesses in children. Neurosurg Focus 2008;24:E8.

6) Saez-Llorens X. Brain abscess in children. Semin Pediatr Infect Dis 2003;14:108-14.

7) Dehority W, Uchiyama S, Khosravi A, Nizet V. Brain abscess caused by Streptococcus pyogenes in a previously healthy child. J Clin Microbiol 2006;44:4613-5.

8) Fuchs $H$, von Baum $H$, Meth $M$, Wellinghausen $N$, Lindner W, Hummler H. CNS-manifestation of aspergillosis in an extremely lowbirth-weight infant. Eur J Pediatr 2006;165:476-80.

9) Chalstrey S, Pfleiderer AG, Moffat DA. Persisting incidence and mortality of sinogenic cerebral abscess: a continuing reflection of late clinical diagnosis. J R Soc Med 1991;84:193-5.

10) Schliamser SE, Backman $K$, Norrby SR. Intracranial abscesses in adults: an analysis of 54 consecutive cases. Scand J Infect Dis 1988;20:1-9.

11) Chun CH, Johnson JD, Hofstetter M, Raff MJ. Brain abscess. A study of 45 consecutive cases. Medicine (Baltimore) 1986;65:415-31.

12) Kao PT, Tseng HK, Liu CP, Su SC, Lee CM. Brain abscess: clinical analysis of 53 cases. J Microbiol Immunol Infect 2003;36:129-36.

13) Barea LM, Tannhauser M, Rotta NT. An epidemiologic study of headache among children and adolescents of southern Brazil. Cephalalgia 1996;16:545-9.

14) Kalinowska-Nowak A, Garlicki A, Bociaga-Jasik M. Brain abscessmodern diagnostics and therapeutic treatment. Przegl Epidemiol 2009;63:89-95.

15) Finkelstein JA, Christiansen CL, Platt R. Fever in pediatric primary care: occurrence, management, and outcomes. Pediatrics 2000;105:260-6.

16) Seydoux C, Francioli P. Bacterial brain abscesses: factors influencing mortality and sequelae. Clin Infect Dis 1992;15:394-401.

17) Barnes PD, Taylor GA. Imaging of the neonatal central nervous system. Neurosurg Clin N Am 1998;9:17-47.

18) Tattevin P, Bruneel F, Clair B, Lellouche F, de Broucker T, Chevret $S$, et al. Bacterial brain abscesses: a retrospective study of 94 patients admitted to an intensive care unit (1980 to 1999). Am J Med 2003;115:143-6.

19) Yang SY, Zhao CS. Review of 140 patients with brain abscess. Surg Neurol 1993;39:290-6

20) Jansson AK, Enblad P, Sjolin J. Efficacy and safety of cefotaxime in combination with metronidazole for empirical treatment of brain abscess in clinical practice: a retrospective study of 66 consecutive cases. Eur J Clin Microbiol Infect Dis 2004;23:7-14.

21) Cavusoglu H, Kaya RA, Turkmenoglu ON, Colak I, Aydin Y. Brain abscess: analysis of results in a series of 51 patients with a combined surgical and medical approach during an 11-year period. Neurosurg Focus 2008;24:E9.

22) Carpenter J, Stapleton S, Holliman R. Retrospective analysis of 49 cases of brain abscess and review of the literature. Eur J Clin Microbiol Infect Dis 2007;26:1-11.

23) Hakan T, Ceran N, Erdem I, Berkman MZ, Goktas P. Bacterial brain abscesses: an evaluation of 96 cases. J Infect 2006;52:359-66.

24) Lee TJ, Chun JK, Kim KH, Kim KJ, Kim DS. Clinical features of brain abscesses in neonates and children: a single center experience from 1997 to 2006. Korean J Pediatr Infect Dis 2008;15:29-34.

25) Lee YH, Go CJ. Clinical studies of brain abscess in children. J Korean Neurol Assoc 1985;3:210-6. 